\title{
On the automaticity of contingent capture: disruption caused by the attentional blink
}

\author{
Feng Du • Jiaoyan Yang • Yue Yin • Kan Zhang • \\ Richard A. Abrams
}

Published online: 27 February 2013

(C) Psychonomic Society, Inc. 2013

\begin{abstract}
Converging evidence has shown that onset capture can be completely eliminated by the demands of a concurrent task and during the attentional blink. In the present study, we investigated contingent capture during the attentional blink. We found that contingent capture was attenuated, or even completely eliminated, during the "blink" time of the attentional blink. These results indicate that contingent capture requires limited attentional resources.
\end{abstract}

Keywords Attentional blink $\cdot$ Contingent capture $\cdot$ Onset capture $\cdot$ Involuntary orienting

Involuntary capture comes in two distinct types. One is the stimulus-driven capture that occurs when an irrelevant stimulus grabs attention by virtue of its low-level salience. For example, abrupt onsets pull attention to a new object in the absence of voluntary control (Yantis \& Jonides, 1984). The other type of involuntary capture is contingent capture, in which an irrelevant stimulus captures attention because it shares features with the targets (Du \& Abrams, 2012; Folk, Remington, \& Johnston, 1992; Folk, Remington, \& Wright, 1994). For example, Folk, Leber, and Egeth (2002) found that the identification of a uniquely colored target at fixation is often disrupted by a peripheral distractor that matches the target color (referred to as a spatial blink). An fMRI study of the spatial blink showed that attention is drawn to the target-

\footnotetext{
F. Du $(\bowtie) \cdot J$. Yang $\cdot$ Y. Yin $\cdot K$. Zhang

Key Laboratory of Behavorial Science, Institute of Psychology, Chinese Academy of Sciences, Beijing, China 100101

e-mail: duf@psych.ac.cn

J. Yang $\cdot$ Y. Yin

University of the Chinese Academy of Sciences, Beijing, China 100049
}

\section{R. A. Abrams}

Washington University, St. Louis, MO 63130, USA colored distractor, even though it is known to be irrelevant (Serences et al., 2005).

Involuntary capture also has an implication of automaticity. For example, onset capture was believed to be highly automatic (Yantis \& Jonides, 1984). Thus, onset capture should remain intact even while a participant performs a secondary task. However, several studies have challenged that traditional belief by showing that onset capture is susceptible to a concurrent monitoring task (Boot, Brockmole, \& Simons, 2005; Santangelo, Olivetti Belardinelli, \& Spence, 2007).

Studies have shown that search efficiency for an orientation singleton was impaired by the attentional blink (AB), an inability to detect a second target (T2) that appears within $400 \mathrm{~ms}$ of an earlier target (T1). These results indicate that involuntary orienting to a singleton requires attention (Du, Abrams, \& Zhang, 2011; Joseph, Chun, \& Nakayama, 1997). If onset capture is truly automatic, it presumably would not be affected by the AB. However, two recent studies have shown that exogenous cuing by uninformative onsets is completely suppressed by the $\mathrm{AB}$ (Du \& Abrams, 2009; Visser, 2011). Since both abrupt onsets and feature singletons fail to capture attention when attention is intensively engaged in object processing, stimulus-driven capture is not automatic.

Few studies, however, have focused on whether contingent capture is automatic. One study incorporated a contingent-capture task as the second task of a psychological refractory period paradigm to test the impact of tone processing on contingent capture (Brisson, Leblanc, \& Jolicœur, 2009). The study used the N2pc response, an enhanced negativity over posterior scalp electrodes contralateral to attended stimuli, in order to index contingent capture. Brisson et al. found a dramatically attenuated $\mathrm{N} 2 \mathrm{pc}$ in response to a target-color distractor if it appeared $200 \mathrm{~ms}$ after an earlier target, relative to when it appeared $500 \mathrm{~ms}$ after $\mathrm{T} 1$, indicating that contingent capture is 
sensitive to the modulation of attentional load. However, the behavioral measurements of contingent capture (accuracy decrement and RT delay) in their study were not modulated by the T1-T2 interval.

In addition, some researchers have suggested that onset capture is a special case of contingent capture (Folk et al., 1992). Thus, contingent capture might be expected to also be suppressed during the AB. However, a study has revealed important differences between contingent capture and onset capture, suggesting that they may be governed by distinct mechanisms (Du \& Abrams, 2010b). Therefore, whether contingent capture is truly automatic is still unknown. In the present experiments, we used an $\mathrm{AB}$ paradigm to test whether contingent capture is immune to the $A B$, and thus truly automatic.

\section{Experiments $1 \mathrm{~A}$ and $1 \mathrm{~B}$}

Previous studies have reported a complete suppression of onset capture within the first $200 \mathrm{~ms}$ of the AB (Du \& Abrams, 2009; Visser, 2011). Contingent capture would also be expected to be suppressed by the AB if it shares similar mechanisms. In order to test the automaticity of contingent capture, in the present experiments we adopted an $\mathrm{AB}$ paradigm with two important methodological features. First, T1 was a visual instead of an auditory target, as in the Brisson et al. (2009) study, which might increase modality-specific interference to contingent capture. Second, color-matched distractors could appear $94 \mathrm{~ms}$ after $\mathrm{T} 1$, an interval shorter than that used by Brisson et al., and one that is more likely to produce strong suppression of attentional capture (Visser, 2011). These changes provided a challenging situation in which to test the resistance of contingent capture to the $\mathrm{AB}$.

Experiment 1a was designed to examine whether contingent capture is immune to the $\mathrm{AB}$. Experiment $1 \mathrm{~b}$ was a replication of Experiment 1a, except that T1 identification was not required. If the reduction in the contingentcapture effect at lag 1 in Experiment 1a was caused by processing $\mathrm{T} 1$, then there should be no such reduction in Experiment $1 \mathrm{~b}$.

Method

Participants A group of 28 undergraduates participated in Experiment 1a, and another 16 undergraduates participated in Experiment $1 \mathrm{~b}$ for payment. All had normal or correctedto-normal visual acuity and no prior experience.

Apparatus and procedure Stimuli were presented on a 17in. monitor with an $85-\mathrm{Hz}$ refresh rate at a distance of $60 \mathrm{~cm}$. The sequence of events on a trial is illustrated in Fig. 1. Each trial began with a 500-ms presentation of a white fixation cross at the center of the screen, followed by the sequential presentation of 24 uppercase letters also at the center. The letters $\left(1.0^{\circ}\right.$ wide, $1.2^{\circ}$ high $)$ were selected randomly without replacement from the English alphabet, with the exception of "I." Each letter was presented for $35 \mathrm{~ms}$, followed by a 59-ms blank interval, yielding a stimulus onset asynchrony (SOA) of $94 \mathrm{~ms}$.

In Experiment 1a, the sole digit in the sequence served as T1. One half of the participants then were required to report the sole red letter as T2; the other half searched for the sole green letter as T2. Across trials, T2 appeared within the 11th-14th or the 16th-19th frames of the letter sequence. The colors of the remaining letters were randomly chosen from three colors (gray, blue, or purple). The participants reported the target letters by pressing the corresponding key after each trial. In the stream of letters, one of the letters in the 9 th -17 th frames, randomly chosen with equal chances, was surrounded by four pound signs (\#), whose inner edges appeared $4.3^{\circ}$ above, below, to the right of, and to the left of the center of the letter. For one third of the trials, all of the pound signs were gray; on the other trials, one of the pound signs was either red or green (equally likely), and the other three were gray. In the present study, distractors always refers to these pound signs in the periphery. The frame

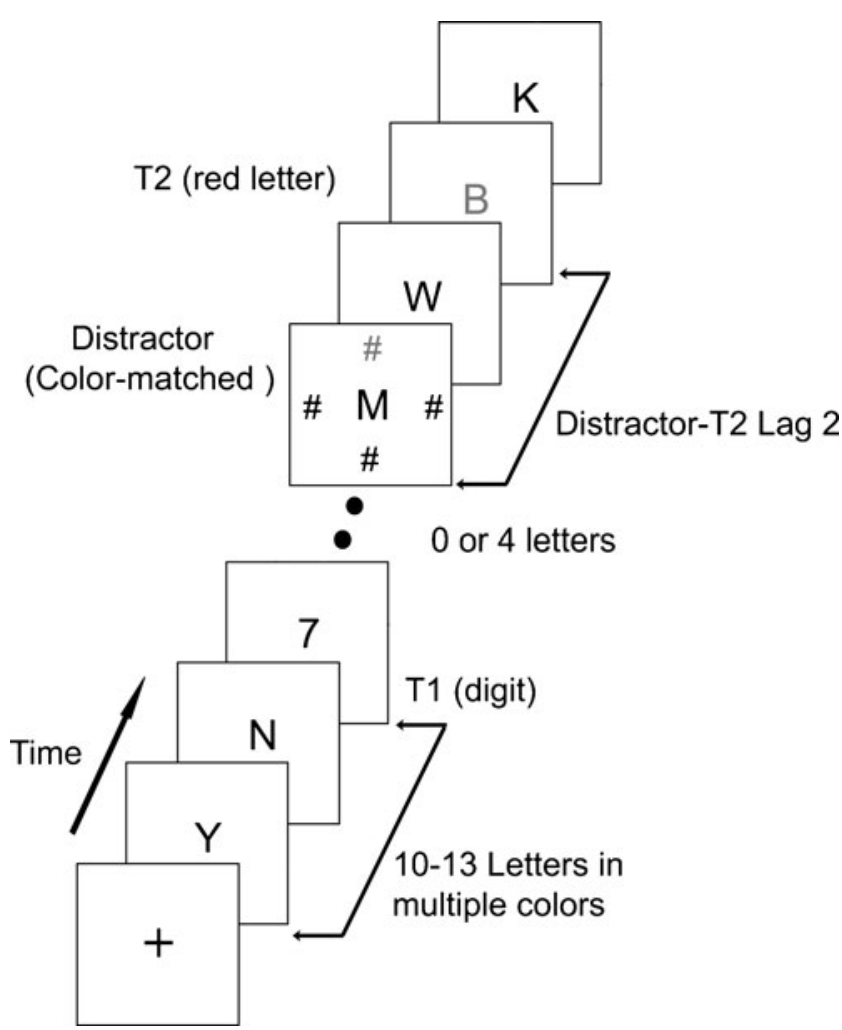

Fig. 1 A schematic representation of the procedure of Experiment 1. Each frame was presented for $35 \mathrm{~ms}$ and followed by a blank interval of $59 \mathrm{~ms}$ 
Table 1 Accuracy of the first target (T1) identification as a function of T1-distractor lag and distractor condition in Experiment 1a

\begin{tabular}{llll}
\hline & \multicolumn{2}{l}{ Distractor Condition } \\
\cline { 2 - 4 } $\begin{array}{l}\text { T1-Distractor } \\
\text { Lag }\end{array}$ & Color-Matched & Color-Unmatched & Gray \\
\hline Lag 1 & $0.905(0.082)$ & $0.948(0.066)$ & $0.943(0.075)$ \\
Lag 5 & $0.959(0.039)$ & $0.946(0.061)$ & $0.959(0.058)$ \\
\hline
\end{tabular}

containing the colored distractors could appear right after $\mathrm{T} 1$ (T1-distractor lag of 1) or in the 5th frame after T1 (T1distractor lag of 5), but it always appeared two frames before T2 (distractor-T2 lag of 2). Thus, T2 appeared either three or seven frames after $\mathrm{T} 1$.

Experiment $1 \mathrm{~b}$ was identical to Experiment $1 \mathrm{a}$, except that the participants were only asked to report $\mathrm{T} 2$.

Design Each trial was in one of three distractor conditions: (1) The four pound signs could be all gray (gray); (2) one pound sign could match the target in color (color matched); or (3) one pound sign was the complementary, nontarget color (color unmatched). Each block contained 20 replications of each combination of the three distractor conditions and two intertarget lags, for a total of 120 trials. We presented 15 practice trials before the test and a brief break after every 40 trials.

\section{Results}

Experiment $1 a$ The mean accuracies for $\mathrm{T} 1$ are listed in Table 1. A repeated measures analysis of variance (ANOVA) showed no main effect of distractor condition, $F(2,54)=1.369, p>.05$, but we did find a main effect of T1-distractor lag, $F(1,27)=9.017, p=.006, \eta_{\mathrm{p}}{ }^{2}=$ .25 , with lower $\mathrm{T} 1$ accuracy at the short lag, and a significant interaction of distractor condition and lag, $F(2,54)=5.521, p=.007, \eta_{\mathrm{p}}{ }^{2}=.17$, with the lowest T1 accuracy occurring when a color-matched distractor appeared at a T1-distractor lag of 1. This shows that color-matched distractors involuntarily captured attention, interfering with $\mathrm{T} 1$ processing.

$\mathrm{T} 2$ accuracy given correct identification of $\mathrm{T} 1$ is shown in Fig. 2a, where it is plotted as a function of lag and distractor condition. A main effect of lag is apparent, $F(1$, 27) $=83.537, p<.001, \eta_{\mathrm{p}}{ }^{2}=.756$, with performance being poorer at $\mathrm{T} 1$-distractor lag 1 than at T1-distractor lag 5. This reveals a large $\mathrm{AB}$ effect. The main effect of distractor condition was also significant, $F(2,54)=27.26$, $p<.001, \eta_{\mathrm{p}}{ }^{2}=.50$, with the lowest T2 accuracy occurring in the color-matched condition. This shows a large contingent-capture effect. Importantly, the interaction between lag and distractor condition was also significant, $F(2,54)=3.324, p=.043, \eta_{\mathrm{p}}{ }^{2}=.11$, with less impairment of T2 performance when color-matched distractors appeared at lag 1, relative to lag 5. Thus, this interaction indicates a greater contingent-capture effect for a long as opposed to a short lag after T1. Post hoc tests showed that the color-matched distractors produced significant capture at both lag $5, F(2,54)=33.337, p<.001, \eta_{\mathrm{p}}{ }^{2}=.553$, and lag $1, F(2,54)=3.47, p=.038, \eta_{\mathrm{p}}{ }^{2}=.114$. Thus, although contingent capture was present at both lags, it was significantly attenuated when the peripheral distractor display was influenced by the AB.

Experiment $1 b$ Since there was no $\mathrm{T} 1$ in Experiment $1 \mathrm{~b}$, we have plotted T2 accuracy as a function of lag and distractor condition in Fig. 2b. There we found no main effect of lag, $F(1,15)=1, p=.333, \eta_{\mathrm{p}}{ }^{2}=.063$. Thus, the $\mathrm{AB}$ effect in Experiment 1a was truly due to processing T1. As expected, the main effect of distractor condition was significant, $F(2,30)=18.946, p<.001, \eta_{\mathrm{p}}{ }^{2}=.558$, with the lowest T2 accuracy in the color-matched condition at both lags. This shows a large contingent-capture effect. Most importantly, the interaction between lag and distractor condition was not significant, $F(2,30)=1.19$, $p=.318, \eta_{\mathrm{p}}{ }^{2}=.074$, showing that the contingent-capture effect at lag 5 was the same as at lag 1 .
Fig. 2 Accuracy of secondtarget (T2) identification as a function of T1-distractor lag and distractor condition in Experiments 1a (panel a) and $1 \mathrm{~b}$ (panel b). In Experiment 1b, the participants were not required to report $\mathrm{T} 1$. The error bars represent the withinsubjects standard errors
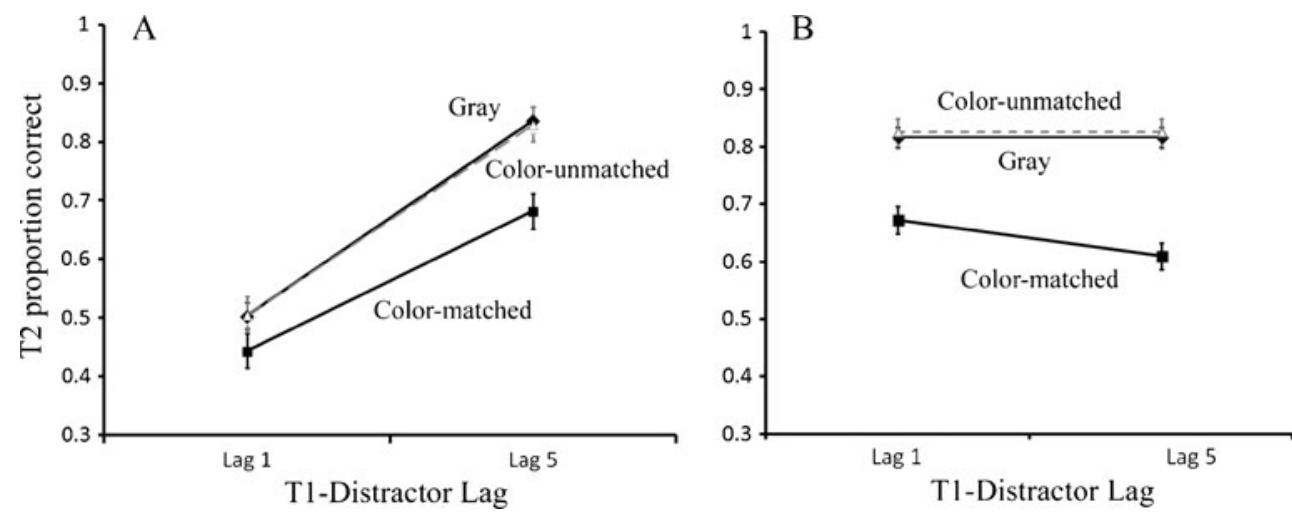
Table 2 Accuracy of the first target (T1) identification as a function of T1-distractor lag and distractor condition in Experiment 2a

\begin{tabular}{llll}
\hline & \multicolumn{2}{l}{ Distractor Condition } \\
\cline { 2 - 4 } T1-Distractor & Color-Matched & Color-Unmatched & Gray \\
Lag & & & \\
\hline Lag 1 & $0.941(0.062)$ & $0.951(0.053)$ & $0.948(0.068)$ \\
Lag 2 & $0.930(0.069)$ & $0.960(0.052)$ & $0.950(0.051)$ \\
Lag 5 & $0.949(0.050)$ & $0.956(0.071)$ & $0.967(0.049)$ \\
\hline
\end{tabular}

\section{Experiments 2A and 2B}

Experiment 1 showed that the $\mathrm{AB}$ attenuated contingent capture. However, contingent capture seemed to persist even at the shortest lag during the $\mathrm{AB}$, which is in contrast to a complete suppression of onset capture within the first $200 \mathrm{~ms}$ of the AB (Du \& Abrams, 2009; Visser, 2011). Three possible accounts could explain why the $\mathrm{AB}$ would not have completely eliminated contingent capture in Experiment 1. One possibility is that contingent capture is more resilient to the $\mathrm{AB}$ than is onset capture. An alternative explanation would be that "lag-1 sparing" might help contingent capture survive the disruption of the $\mathrm{AB}$. To rule out this possibility, in Experiment 2a we examined T1distractor lags of 1,2, and 5. Contingent capture would most likely be eliminated at lag 2 if lag-1 sparing did occur in Experiment 1a. Finally, we considered a third possibility, that the SOA in Experiment 1a was not short enough to completely eliminate contingent capture. Theoretically, if contingent capture is reduced by the $\mathrm{AB}$, complete suppression of contingent capture could be observed with a shorter SOA. Thus, in the present experiment we shortened the SOA to examine this possibility.

\section{Method}

Participants A group of 31 undergraduates participated in Experiment 2a, and another 18 undergraduates participated in Experiment 2b for payment. All had normal or correctedto-normal visual acuity and no prior experience. One participant in Experiment 2a was excluded from the data analysis because his T1 accuracy was lower than $85 \%$.

Apparatus, procedure, and design Experiment 2a was the same as Experiment 1a, with two exceptions. First, each letter was presented for $35 \mathrm{~ms}$, followed by a $47-\mathrm{ms}$ blank interval, yielding an SOA of $82 \mathrm{~ms}(12 \mathrm{~ms}$ shorter than in Exp. 1a). Second, the frame containing the colored distractor could appear right after T1 (T1-distractor lag of 1 ) or in the 2 nd or the 5 th frame after $\mathrm{T} 1$ (T1-distractor lags of 2 and 5, respectively). Each combination of three distractor conditions and three lags was presented 24 times, for a total of 216 trials. Also, 27 practice trials were presented before the test, and a brief break after every 54 trials.

Experiment $2 \mathrm{~b}$ was identical to Experiment 2a, except that the participants were only asked to report T2.

\section{Results}

Experiment $2 a$ The mean accuracies for $\mathrm{T} 1$ are listed in Table 2. In a repeated measures ANOVA, we found no significant effects, all $p \mathrm{~s}>.10$.

$\mathrm{T} 2$ accuracy given correct identification of $\mathrm{T} 1$ is plotted as a function of lag and distractor condition in Fig. 3a. There we can see a main effect of lag, $F(2,58)=144.874, p<.001, \eta_{\mathrm{p}}{ }^{2}=$ .833 , with performance being lowest at $\mathrm{T} 1$-distractor lag 1 and highest at lag 5. This confirms a large $\mathrm{AB}$ effect and the absence of lag-1 sparing. The main effect of distractor condition was significant, $F(2,58)=12.203, p<.001, \eta_{\mathrm{p}}{ }^{2}=.296$, with the lowest T2 accuracy in the color-matched condition. Importantly, the interaction between lag and distractor condition was significant, $F(4,116)=2.894, p=.025, \eta_{\mathrm{p}}{ }^{2}=.091$, due to the fact that color-matched distractors caused the least impairment of $\mathrm{T} 2$ performance at lag 1 and the highest impairment at lag 5. Thus, a greater contingent-capture effect occurred at a long than at a short lag after T1. Post hoc
Fig. 3 Accuracy of secondtarget (T2) identification as a function of T1-distractor lag and distractor condition in Experiments 2a (panel a) and $2 b$ (panel b). In Experiment 2b, the participants were not required to report $\mathrm{T} 1$. The error bars represent the withinsubjects standard errors
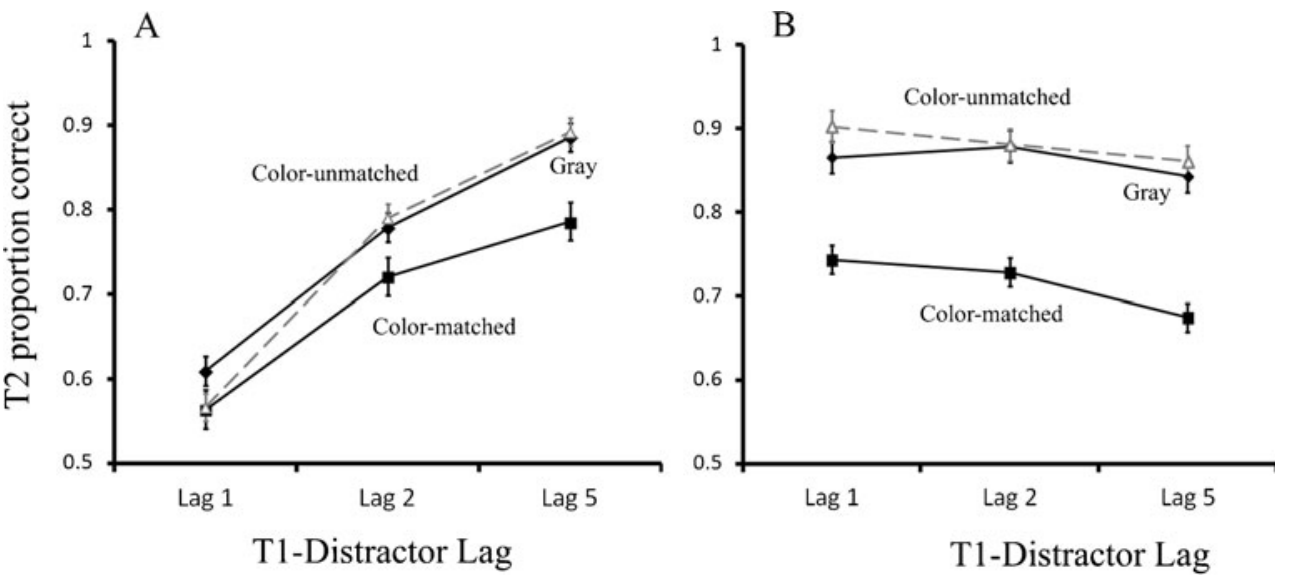
comparisons showed that the color-matched distractors produced the greatest capture at T1-distractor lag 5, $F(2$, 58) $=15.383, p<.001, \eta_{\mathrm{p}}{ }^{2}=.347$, and less capture at lag $2, F(2,58)=5.117, p=.009, \eta_{\mathrm{p}}{ }^{2}=.150$, but no significant capture occurred at lag $1, F(2,58)=1.889, p=$ $.160, \eta_{\mathrm{p}}{ }^{2}=.061$. Thus, contingent capture can be largely suppressed by the AB.

Experiment $2 b \mathrm{~T} 2$ accuracy in Experiment $2 \mathrm{~b}$ is plotted as a function of lag and distractor condition in Fig. 3b. We found a main effect of lag, $F(2,34)=4.537, p=.018, \eta_{\mathrm{p}}{ }^{2}=.211$, with lower T2 performance at lag 5 than at lag 1 . Thus, the $\mathrm{AB}$ effect in Experiment 2a was truly due to processing $\mathrm{T} 1$. As expected, the main effect of distractor condition was significant, $F(2,34)=21.937, p<.001, \eta_{\mathrm{p}}{ }^{2}=.563$, with the lowest T2 accuracy in the color-matched condition. This replicated the results of Experiment 1b. Importantly, the interaction between lag and distractor condition was not significant, $F(4,68)<1$, showing that the contingent capture was the same at all three lags.

\section{Experiment 3}

Experiment 3 was designed to examine whether colormatched distractors induce involuntary shifts of attention during the $\mathrm{AB}$ by using identity intrusion techniques. For the present experiment, we replaced one distractor (\#s in the earlier experiments) with a letter that could be either congruent or incongruent with $\mathrm{T} 2$. If attention were involuntarily pulled to the color-matched distractors, identification of T2 should reflect the identity of the distracting letter. Thus, colormatched distractors should lead to better T2 performance in the congruent than in the incongruent condition. Such a result would bolster the conclusion that color-matched distractors produce the contingent capture of spatial attention.

\section{Method}

Participants A group of 20 new undergraduate students participated in a half-hour experiment for payment. All had normal or corrected-to-normal visual acuity.

Apparatus and procedure The stimuli and procedure were the same as in Experiment 1a, with three exceptions. First, for half of the participants, T1 was defined as the first red digit or letter in the sequence. For the other half, T1 was defined as the first green digit or letter. Second, T2 was a red or green letter that was in the same color as T1, randomly selected from four possibilities (T, L, H, and S). This guaranteed that the same attentional control settings would be in place for the two tasks. Finally, instead of four pound signs in the distractor display, three of the elements were pound signs, and the fourth was a letter that was drawn from the four possible $\mathrm{T} 2$ letters.

Design Two distractor-T2 congruency conditions were defined for this experiment: On one half of the trials, the sole distractor letter in the periphery was the same letter as T2 (congruent), and on the other half of the trials, the sole peripheral distractor letter differed from T2 (incongruent). We also used three distractor conditions: (1) The three pound signs and one letter were all gray (gray); (2) the sole distractor letter matched the target color (color-matched) and the pound signs were gray; or (3) the sole distractor letter was the complementary, nontarget color (colorunmatched), and the pound signs were gray. Each combination of the three distractor conditions, two conditions of distractor-T2 congruency, and two lags was presented 20 times, yielding a total of 240 trials. The experiment began with 18 practice trials, and participants had a brief break after every 60 trials.

\section{Results}

The mean accuracies for $\mathrm{T} 1$ identification are shown in Table 3. An ANOVA showed neither main effects nor an interaction, all $F_{\mathrm{s}}<1.5, p \mathrm{~s}>.235$.

$\mathrm{T} 2$ accuracy given correct identification of $\mathrm{T} 1$ is plotted as a function of distractor-T2 congruency, T1-distractor lag, and distractor condition in Fig. 4. There we can see a main effect of lag, $F(1,19)=48.598, p<.001, \eta_{\mathrm{p}}^{2}=.719$, with performance being poorer at lag 1 than at lag 5, indicating a large $\mathrm{AB}$ effect. The main effect of distractor condition was significant, $F(2,38)=20.169, p<.001, \eta_{\mathrm{p}}{ }^{2}=.515$, with the lowest T2 accuracy in the color-matched condition at both lags. The interaction between lag and distractor condition was also significant, $F(2,38)=6.298, p=.004, \eta_{\mathrm{p}}{ }^{2}=.249$, revealing attenuated contingent capture at lag 1 as compared with lag 5.

In addition, some important new findings emerged. First, the main effect of distractor-T2 congruency was significant, $F(1,19)=19.249, p<.001, \eta_{\mathrm{p}}{ }^{2}=.503$, with higher $\mathrm{T} 2$ performance in the congruent than in the incongruent condition. Second, we found an interaction between distractor condition and distractor-T2 congruency, $F(2,38)=17.652$,

Table 3 Accuracy of the first target (T1) identification as a function of T1-distractor lag and distractor condition in Experiment 3

\begin{tabular}{llll}
\hline & \multicolumn{2}{l}{ Distractor Condition } \\
\cline { 2 - 4 } T1-Distractor & Color-Matched & Color-Unmatched & Gray \\
Lag & & & \\
\hline Lag 1 & $0.897(0.071)$ & $0.895(0.074)$ & $0.897(0.073)$ \\
Lag 5 & $0.927(0.052)$ & $0.905(0.066)$ & $0.895(0.047)$ \\
\hline
\end{tabular}




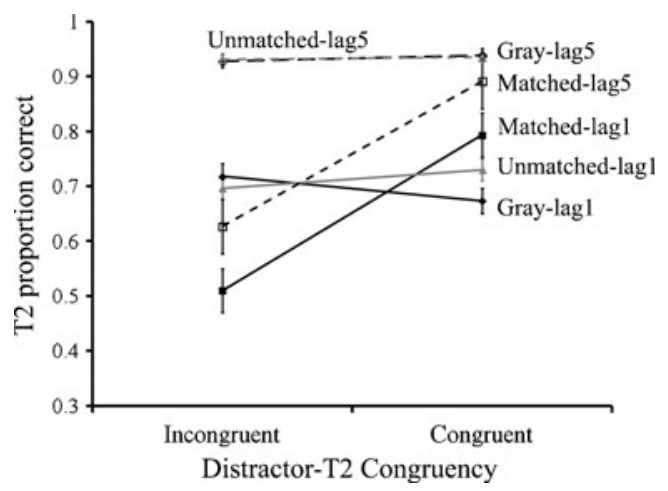

Fig. 4 Accuracy of second-target (T2) identification as a function of distractor-T2 identity condition, T1-distractor lag, and distractor condition in Experiment 3. The error bars represent the within-subjects standard errors

$p<.001, \eta_{\mathrm{p}}{ }^{2}=.482$, showing that the congruency effect was only observed in the color-matched condition. These findings demonstrate that the color-matched distractors captured spatial attention involuntarily, resulting in processing of the identity of the distractors and a large congruency effect. Finally, no interaction between T1-distractor lag and congruency emerged, $F(2,38)<1, p=.939$, nor was there a three-way interaction, $F(2,38)=1.274, p=.291, \eta_{\mathrm{p}}{ }^{2}=.063$. These results indicate that the congruency effect was unaffected by the AB.

\section{General discussion}

The present experiments showed that the attentional blink suppressed the capturing effect of a target-color distractor presented shortly after $\mathrm{T} 1$, indicating that contingent capture is impaired by the $\mathrm{AB}$ and requires attentional resources. This result is consistent with previous findings that the N2pc associated with color-matched distractors was attenuated during psychological refractory period tasks (Brisson et al., 2009), but differs from the behavioral results of that same study, thus helping to clarify that a behavioral index is sensitive enough to detect the suppressive effect of $A B$ on contingent capture.

The present findings differ somewhat from a previous study showing that a peripheral color-matched distractor did not capture attention when a central color-matched distractor appeared before the peripheral one (Folk, Ester, \& Troemel, 2009). Folk et al. (2009) reported that the suppression lasted at least for $300 \mathrm{~ms}$, which is much longer than the suppression that we reported here. The discrepancy between the two studies is probably due to the distinctly different attentional control settings required in the two studies. In the present study, we aimed to examine whether voluntary processing of T1 would reduce contingent capture, whereas Folk et al. (2009) tested whether earlier contingent capture by a central color-matched distractor would disrupt subsequent contingent capture by a peripheral color-matched distractor. Naturally, the two studies differed in their tasks. The present study required participants to report two consecutive targets in the RSVP stream. Thus, the participants would not completely shut off the attentional gate after detecting T1, and peripheral distractors could be processed to a high level (Luck, Vogel, \& Shapiro, 1996), which might explain small contingent capture occurring as early as $94 \mathrm{~ms}$ after T1. However, when the participants in the Folk et al. (2009) study were tuned to detect only one specifically colored target, a central color-matched distractor might simply trigger involuntary selection of that distractor and shut off the attentional gate. Thus, whatever came after this central color-matched distractor, including peripheral color-matched distractors, would be suppressed until participants realized the mistake and reopened their attentional gate.

Researchers have suggested that onset capture may be a special case of contingent capture (Folk et al., 1992). However, a study has shown that contingent capture was biased to the left visual hemifield, whereas onset capture was symmetric, suggesting that contingent capture may be fundamentally different from onset capture (Du \& Abrams, 2010b). In the present study, we showed that contingent capture could be largely removed during the "blink" time of the AB. This finding is consistent with earlier studies that have examined onset capture using the $\mathrm{AB}$ paradigm (Du \& Abrams, 2009; Visser, 2011). In those studies, when a distractor suddenly onset $100 \mathrm{~ms}$ after $\mathrm{T} 1$, onset capture was entirely eliminated. Thus, contingent capture appears to share part of its underlying mechanism with onset capture. A possible candidate is the spatial-orienting component. Note that although a color-matched distractor may capture attention in a way quite different from an abrupt onset (Du \& Abrams, 2008), the behavioral consequences of the two types of capture are the same: involuntary orienting of attention. This speculation is consistent with previous fMRI studies showing that onset capture and contingent capture recruit generally overlapping neural networks for spatial orienting (Serences et al., 2005). Furthermore, studies have shown that spatial attention is "frozen" for the first $100 \mathrm{~ms}$ of the $\mathrm{AB}$ ( $\mathrm{Du} \&$ Abrams, 2010a). Event-related potential studies have also shown that voluntary orienting during the $\mathrm{AB}$ is associated with a decreased N2pc magnitude, further showing the susceptibility of these mechanisms to disruption (Dell'Acqua, Sessa, Jolicœur, \& Robitaille, 2006).

The present study has shown that contingent capture is suppressed $82 \mathrm{~ms}$ after T1, but recovers by $164 \mathrm{~ms}$ after T1 (it was present $94 \mathrm{~ms}$ after T1 in Exp. 1a). This is slightly different from the temporal course of onset capture in the AB. The suppression of onset capture can be extended to 
200 ms after T1 (Du \& Abrams, 2009; Visser, 2011). This difference seems to suggest that contingent capture is quantitatively more resilient to disruption by the $\mathrm{AB}$ than is onset capture, if these studies are comparable. However, the difference needs further examination in a single experimental setting.

Regardless of the explanation for disruption of contingent capture during the $\mathrm{AB}$, it is also clear that contingent capture was attenuated by the $\mathrm{AB}$, thus revealing that it requires at least some limited attentional resources.

Author Note This study was supported by grants from the National Natural Science Foundation of China (Grant Nos. 31200766 and 91124003) and the Scientific Foundation of Institute of Psychology, Chinese Academy of Sciences (Grant No. Y1CX212005).

\section{References}

Boot, W. R., Brockmole, J. R., \& Simons, D. J. (2005). Attention capture is modulated in dual-task situations. Psychonomic Bulletin \& Review, 12, 662-668. doi:10.3758/BF03196755

Brisson, B., Leblanc, E., \& Jolicœur, P. (2009). Contingent capture of visual-spatial attention depends on capacity-limited central mechanisms: Evidence from human electrophysiology and the psychological refractory period. Biological Psychology, 80, 218-225.

Dell'Acqua, R., Sessa, P., Jolicœur, P., \& Robitaille, N. (2006). Spatial attention freezes during the attention blink. Psychophysiology, 43, 394-400. doi:10.1111/j.1469-8986.2006.00411.x

Du, F., \& Abrams, R. A. (2008). Synergy of stimulus-driven salience and goal-directed prioritization: Evidence from the spatial blink. Perception \& Psychophysics, 70, 1489-1503. doi:10.3758/ PP.70.8.1489

Du, F., \& Abrams, R. A. (2009). Onset capture requires attention. Psychonomic Bulletin \& Review, 16, 537-541. doi:10.3758/ PBR.16.3.537

Du, F., \& Abrams, R. A. (2010a). Endogenous orienting is reduced during the attentional blink. Experimental Brain Research, 205, $115-121$.
Du, F., \& Abrams, R. A. (2010b). Visual field asymmetry in attentional capture. Brain and Cognition, 72, 310-316.

Du, F., \& Abrams, R. A. (2012). Out of control: Attentional selection for orientation is thwarted by properties of the underlying neural mechanisms. Cognition, 124, 361-366.

Du, F., Abrams, R. A., \& Zhang, K. (2011). Spatial distribution of the attentional blink. Frontiers in Cognition, 2, 360.

Folk, C. L., Ester, E. F., \& Troemel, K. (2009). How to keep attention from straying: Get engaged! Psychonomic Bulletin \& Review, 16, 127-132. doi:10.3758/PBR.16.1.127

Folk, C. L., Leber, A. B., \& Egeth, H. E. (2002). Made you blink! Contingent attentional capture produces a spatial blink. Perception \& Psychophysics, 64, 741-753. doi:10.3758/BF03194741

Folk, C. L., Remington, R. W., \& Johnston, J. C. (1992). Involuntary covert orienting is contingent on attentional control settings. Journal of Experimental Psychology: Human Perception and Performance, 18, 1030-1044. doi:10.1037/0096-1523.18.4.1030

Folk, C. L., Remington, R. W., \& Wright, J. H. (1994). The structure of attentional control: Contingent attentional capture by apparent motion, abrupt onset, and color. Journal of Experimental Psychology: Human Perception and Performance, 20, 317-329. doi:10.1037/0096-1523.20.2.317

Joseph, J. S., Chun, M. M., \& Nakayama, K. (1997). Attentional requirements in a "preattentive" feature search task. Nature, 387, 805-807.

Luck, S. J., Vogel, E. K., \& Shapiro, K. L. (1996). Word meanings can be accessed but not reported during the attentional blink. Nature, 383, 616-618. doi:10.1038/383616a0

Santangelo, V., Olivetti Belardinelli, M., \& Spence, C. (2007). The suppression of reflexive visual and auditory orienting when attention is otherwise engaged. Journal of Experimental Psychology: Human Perception and Performance, 33, 137-148. doi:10.1037/ 0096-1523.33.1.137

Serences, J. T., Shomstein, S., Leber, A. B., Golay, X., Egeth, H. E., \& Yantis, S. (2005). Coordination of voluntary and stimulus-driven attentional control in human cortex. Psychological Science, 16, $114-122$.

Visser, T. A. W. (2011). A re-examination of the impact of object processing on shifts of spatial attention. Attention, Perception, \& Psychophysics, 73, 688-694. doi:10.3758/s13414-010-0058-6

Yantis, S., \& Jonides, J. (1984). Abrupt visual onsets and selective attention: Evidence from visual search. Journal of Experimental Psychology: Human Perception and Performance, 10, 601-621. doi:10.1037/0096-1523.10.5.601 